Review Article

\title{
Autophagy Induced by ROS Aggravates Testis Oxidative Damage in Diabetes via Breaking the Feedforward Loop Linking p62 and Nrf2
}

\author{
Yuanyuan Tian, ${ }^{1}$ Wei Song, ${ }^{1}$ Dongsheng Xu, ${ }^{1}$ Xiao Chen, ${ }^{1}$ Xiaojiao Li, ${ }^{2}$ \\ and Yuguang Zhao ${ }^{1,2}$ \\ ${ }^{1}$ Cancer Center, The First Hospital of Jilin University, Changchun, Jilin 130021, China \\ ${ }^{2}$ Phase I Clinical Trials Unit, The First Hospital of Jilin University, Changchun, Jilin 130021, China \\ Correspondence should be addressed to Yuguang Zhao; zhaoyuguang@jlu.edu.cn
}

Received 15 March 2020; Revised 26 April 2020; Accepted 29 April 2020; Published 19 May 2020

Academic Editor: Gianna Ferretti

Copyright (C) 2020 Yuanyuan Tian et al. This is an open access article distributed under the Creative Commons Attribution License, which permits unrestricted use, distribution, and reproduction in any medium, provided the original work is properly cited.

\begin{abstract}
Testicular dysfunction due to hyperglycemia is the main cause of infertility in diabetic men. Over the years, in order to solve this growing problem, a lot of research has been done and a variety of treatments have been created, but so far, there is no safe, effective, and practical method to prevent male infertility caused by diabetes. In this review, we emphasize the male infertility mechanism caused by diabetes from the effects of oxidative stress and autophagy on the function of testes via the PI3K/Akt/mTOR signaling pathway, and we highlight that oxidative stress-induced autophagy breaks the feedforward loop linking Nrf2 and p62 and promotes oxidative damage in diabetic testes.
\end{abstract}

\section{Introduction}

As a multifactorial disease characterized by hyperglycemia, the incidence of diabetes has been increasing in the past decades [1]. According to the latest data, 463 million adults currently have diabetes. If adequate action is not taken to address the epidemic, 578 million people will have diabetes by 2030 . By 2045 , that number will jump to a staggering 700 million [2]. It is well known that diabetes can negatively affect the fertility of women and men, and studies have shown that diabetes can cause cellular abnormalities in reproductive organs [3-6]. Testicular dysfunction is a major complication of diabetic patients, especially those at reproductive age, and its incidence is increasing worldwide [7-9]. In male reproductive organs, testes are most vulnerable to hyperglycemia [9]. Animal studies using the diabetic rodent models have shown that diabetes can damage the epididymis of rodents, influence the quality of semen, and thus impair reproductive function [10-13]. Previous clinical studies have confirmed that sperm count, motility, and morphology in diabetic patients are sig- nificantly worse compared to the control group [14-16]. In addition, previous studies have demonstrated that diabetic rats have reduced testicular weight [17], abnormal tissue structure of seminiferous epithelium, vacuolization of Sertoli cells (SCs) [18], and disruption of the blood-testis barrier (BTB) [19]. Therefore, it is of great significance to study the mechanism of testicular dysfunction in diabetic patients and find an effective way to prevent male infertility.

In the pathogenesis of male infertility in diabetes, reactive oxygen species (ROS) play a vital role [20]. And under a variety of pathological conditions, the relative excessive accumulation of ROS can induce autophagy [21-24]. In previous animal model studies, it has also been confirmed that in the testis, excessive production of ROS can induce autophagy [25-27]. Therefore, in this review, we speculate that in diabetes, excessive production of ROS can induce autophagy in the testis. A series of studies have confirmed that abnormal autophagy can cause abnormalities in the complex and highly ordered sperm cell differentiation process, such as acrosome biogenesis and sperm differentiation defects $[28$, 
29], decreased serum testosterone levels [30], and SC apoptosis and BTB damage [26, 31]. The phosphatidylinositol 3kinase $(\mathrm{PI} 3 \mathrm{~K}) /$ protein kinase $\mathrm{B}(\mathrm{Akt}) /$ mammalian target of rapamycin (mTOR) signaling pathway is a target of oxidative stress [32]. Furthermore, the PI3K/Akt/mTOR signaling pathway is one of the most vital regulators of autophagy and its activation promotes spermatogenesis [33-35]. In this review, we mainly elaborate that autophagy induced by oxidative stress via the PI3K/Akt/mTOR signaling pathway accelerates the oxidative stress in the testis, and we highlight that autophagy induced by oxidative stress breaks the feedforward loop linking Nrf2 and p62 and aggravates oxidative damage in diabetic testes.

\section{Diabetes and Male Infertility}

Diabetes mellitus (DM) is a multifactorial disease characterized by hyperglycemia. In the past few decades, a lot of work has been done and a variety of treatments have been developed to address this growing problem; however, even today, the prevalence and incidence of diabetes continues to rise sharply. And the incidence of type 1 diabetes (T1D) has been increasing worldwide [36]. At the same time, as the incidence is increasing, the age of onset is also getting younger. According to studies evaluating temporal trends, the incidence of childhood-onset T1D has increased around the world, with an average relative growth of 3\%-4\% per calendar year [37]. This has many implications for the risk of complications. The increased incidence of diabetes in young people is of great concern because it may affect the reproductive function of more men during their active reproductive age.

\section{Hyperglycemia Inducing the Production of ROS}

Diabetes is characterized by a hyperglycemic state, and the elevating level of oxidative stress directly induced by hyperglycemia plays a crucial role in male infertility [38] (Figure 1). Hyperglycemia has been shown to cause oxidative stress through hydroxyl radicals generated by the autoxidation of glucose [39, 40]. Previous investigations have demonstrated that accumulation of advanced glycation end products (AGEs) produced by nonenzymatic reactions between sugar and amino groups of proteins under hyperglycemic conditions and their receptors (RAGE) plays a crucial role in the development of diabetes-induced complications, including the pathogenesis of diabetes-induced male reproductive damage, which activates oxidative stress and increases the generation of ROS $[19,41,42]$. Through semiquantitative analysis of immunostaining of sperm from the diabetic and nondiabetic males, Mallidis et al. also found that in diabetic male sperm samples, the majority of sperm (>60\%) expressed RAGE, which is approximately three times greater than that seen in samples from nondiabetic men [43]. Therefore, in patients with diabetes, excessive hydroxyl radicals and AGEs induced by hyperglycemia can further increase the production of ROS, leading to an increase in oxidative stress and aggravation of sperm loss.

\section{The Role of ROS in Testicular Functions}

As one of the messengers that affect sperm function during the process of sperm from the testis to the oocyte, low levels of ROS can regulate sperm function, promote sperm capacitation, and regulate sperm maturation [44]. Numerous studies have shown that low levels of ROS are essential in the process of obtaining fertilization in sperm. Aitken et al. first proposed the idea that low levels of ROS can regulate the physiological functions of sperm and found that the ability of sperm to bind the zona pellucida can be enhanced by low levels of ROS [45]. Adding low concentration of hydrogen peroxide $\left(\mathrm{H}_{2} \mathrm{O}_{2}\right)$ can stimulate sperm capacitation, overactivation, acrosome reaction, and oocyte fusion, which has also been confirmed by studies [46, 47]. ROS other than $\mathrm{H}_{2} \mathrm{O}_{2}$ such as nitric oxide and superoxide anion have also been shown to promote sperm capacitation and acrosome reaction $[48,49]$. However, human sperm is particularly vulnerable to oxidative damage, and an imbalance between the production of ROS and the antioxidant capacity of sperm will lead to male infertility [50-52]. It has been accepted that spermatozoa are vulnerable to oxidative damage because their plasma membranes contain large amounts of polyunsaturated fatty acids (PUFA) and have low concentrations of scavenging enzymes in their cytoplasm [13,53,54]. When levels are excessive, ROS attack PUFA in the sperm plasma membrane, leading to lipid peroxidation [55]. A large number of studies have shown that long-term hyperglycemia increases the production of ROS in testicular tissues in patients with diabetes and plays a key role in male testicular dysfunction [6, 56-58] (Figure 1).

4.1. Imbalance of Oxidation and Antioxidation in Testes Leads to Male Infertility. Among diabetics, oxidative imbalance is a key hallmark in testes. Hyperglycemia induces the germ cells and somatic cells in the testes to produce excessive ROS $[19,59]$, which overwhelms the endogenous ROS scavenging systems $[60,61]$. Oxidative stress further causes damage to many macromolecules and disrupts their functions, such as lipid peroxidation, protein modifications, and DNA damage [62-64] (Figure 1). And the sperm cell membrane damage induced by oxidative stress may be one of the reasons for infertility $[52,65]$. It has been proved that ROS attacks not only the fluidity of the sperm plasma membrane but also the integrity of DNA in the sperm nucleus [66]. Agarwal et al. have found that the level of apoptosis of mature sperm in infertile patients is significantly higher than that in normal donors in the control group, indicating that DNA damage caused by ROS may accelerate apoptosis of germ cells, further leading to a significant decrease in sperm count and semen quality [67]. The level of DNA oxidation in infertile men's sperm is higher than that in fertile men's sperm, which further proves that the excessive production of ROS causes damage to sperm [68]. Therefore, improving the antioxidative ability of testes may become an effective method for preventing male infertility in diabetic patients.

4.2. PI3K/Akt/mTOR Signaling Pathway as a Central Regulator of Spermatogenesis. The activation of PI3K and 


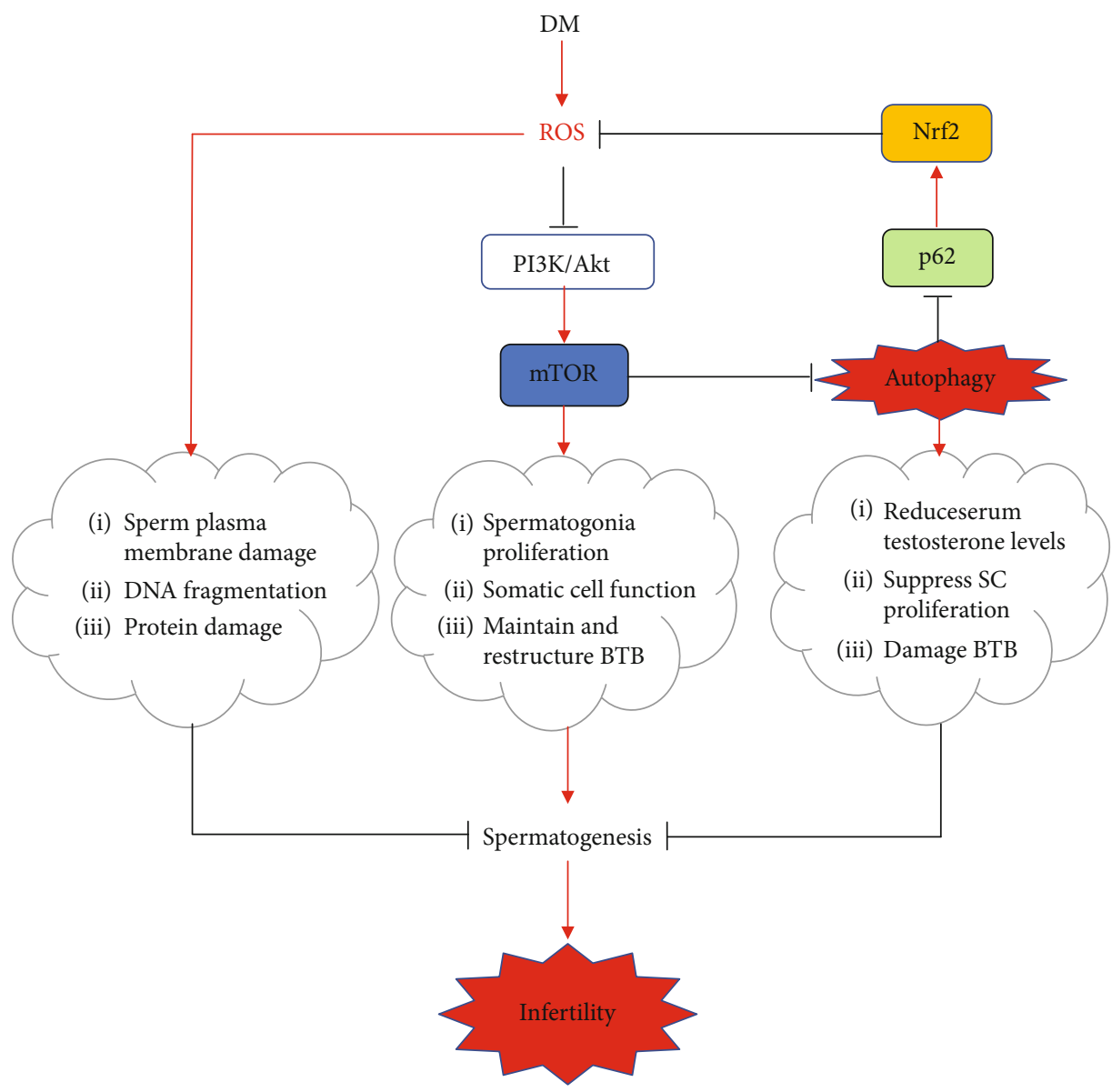

FIGURE 1: Mechanistic illustrations. The increasing formation of ROS in DM can directly cause damage to spermatogenesis via attacking the fluidity of the plasma membrane lipids, protein modifications, and integrity of DNA. And ROS also can induce autophagy by inhibiting mTOR through the PI3K/Akt signaling pathway. Autophagy accelerates the degradation of p62, and then, the Nrf2 activation is suppressed and the oxidative damage is aggravated. Autophagy also directly causes damage to spermatogenesis via reducing serum testosterone levels, suppressing SC proliferation, and damaging BTB. Moreover, mTOR has distinct effects on spermatogenesis via promoting spermatogonia proliferation, maintaining somatic cell function, and restructuring BTB. As a result, the oxidative damage in diabetic testes is further enhanced, thereby promoting the occurrence of infertility.

its downstream target mTOR is beneficial to protein synthesis and cell survival, which has been confirmed by previous studies. Blume et al. have confirmed that the activation of PI3K is essential for stem cell factor-induced spermatogenesis [69]. mTOR, a well-conserved Ser/Thr protein kinase, plays a key role in sensing environmental conditions and regulating cell metabolism [70]. Since the first clinical evidence showed that a 36-year-old male was infertile after taking rapamycin, an mTOR inhibitor, and his sperm analysis showed a dramatic diminution of sperm count, percentage of normal-shaped sperm heads, and sperm motility, a role for mTOR in male reproductive physiology was originally proposed [71]. Studies have verified distinct roles for mTOR in spermatogenesis [72] (Figure 1). Deutsch et al. found that testosterone secretion and sperm count decreased in patients treated with rapamycin, and sperm count and sex hormone levels improved after withdrawing rapamycin [72]. Recent studies have shown that mTOR plays a key role in testicular physiology. mTOR has two different complex forms, mTOR complex 1 (mTORC1) and mTOR complex 2 (mTORC2), which increases the complexity of studying the function of mTOR [73]. mTOR and its associated partner proteins are expressed in both the germ (particularly in spermatogonia) and somatic (Sertoli and Leydig) cells within the testis, with mTORC1 and mTORC2 being differentially present in all these cellular types [74-77]. In spermatogonia, the application of rapamycin caused a decrease in proliferation by blocking the mTOR/p70S6K $(70 \mathrm{kDa}$ ribosomal protein $\mathrm{S} 6$ kinase, a significant downstream effector of $\mathrm{mTOR}$, mediating protein synthesis) pathway, which indicates the role of mTORC1 in maintaining germ cell proliferation [77]. In vivo studies have shown that rapamycin causes atrophy and vacuolation of seminiferous tubules by inhibiting mTORC1, leading to reduced sperm production, which indicates the role of mTORC1 in spermatogenesis [77]. mTORC1-deficient mice show reduced sperm motility, which indicates that mTORC1 can regulate the physiological functions of sperm during the passage of the epididymis, in addition to maintaining germ cell proliferation and spermatogenesis [76]. Studies show that mTOR can directly participate in nutritional support 
for spermatogenesis by controlling glucose consumption and redox balance in SCs [78]. In addition, mTOR also plays a key role in the maintenance and reorganization of $\mathrm{BTB}$, which is very important for maintaining the spermatogenic epithelium circulation $[79,80]$. Although mTOR has been shown to participate in many physiological processes, the role of mTOR and its inhibitors in male reproduction needs more research.

4.3. ROS Inducing Autophagy via Inhibiting the PI3K/Akt Signaling Pathway. Under various stress conditions, such as hyperglycemia, oxidative stress, and starvation, the $\mathrm{PI} 3 \mathrm{~K} / \mathrm{Akt} / \mathrm{mTOR}$ signaling pathway is a typical negative regulatory pathway for autophagy in mammalian germ cells. Studies have reported that overproduction of ROS in longterm hyperglycemic organisms significantly inhibits the PI3K/Akt signaling pathway, thus affecting cell autophagic function (Figure 1). Lin et al. found that ROS induced autophagic cell death by negatively regulating this signaling pathway [81]. Shi et al. also demonstrated that the levels of p-PI3K and p-Akt/t-Akt in diabetic testes cells were significantly downregulated, whereas after treatment with Lycium barbarum polysaccharide, a well-known antioxidant food supplement, p85-PI3K and p-Akt expression were significantly upregulated [82]. Therefore, under hyperglycemic conditions, reducing ROS production can regulate autophagy through a PI3K/Akt-dependent mechanism in testicular tissues. It has also been proved that the increase of oxidative stress inhibits the PI3K/Akt/mTOR pathway, following by the inhibition of the expression of p70S6K, leading to degeneration and malformation of sperm, and affects sperm count, motility, and function in epididymis $[77,83]$.

\section{The Role of Autophagy in Male Testicular Functions}

Autophagy is an intracellular lysosomal degradation pathway and plays a very important role in maintaining intracellular homeostasis [84]. The main role of autophagy is to eliminate intracellular energy resources in nutrient shortage conditions and remove cytotoxic proteins and organelles under stressful situations [85]. Previous studies have shown that autophagy plays an important role in acrosome biogenesis [29] and spermatid differentiation during spermatogenesis [28]. Moderate autophagy maintains homeostasis of organisms and was reported to play a protective role against testicular damage caused by hyperglycemia [85] and hypoxia [86]. However, a series of research studies confirmed that abnormal autophagy is pivotal for male infertility (Figure 1). Leydig cells, as an important part of the testicular stroma, are the main source of androgens [87]. Zhao et al. have shown that autophagy induced by suppressing the Akt-mTOR pathway can inhibit Leydig cells, thereby reducing serum testosterone levels [30]. SCs are essential for spermatogenesis and male fertility, and they coordinate the spermatogenesis process by providing nutrition and an environment conducive to the survival and development of germ cells [88-90]. It has been reported by Duan et al. that in SCs, the mTOR signaling pathway mediated by ROS may be the main pathway to aug- ment autophagy, which causes the suppression of SC proliferation, thus impairing spermatogenesis and fertility [31]. The BTB consisting of tight junctions, adherens junctions, and gap junctions between adjoining SCs plays a key role in the spermatogenesis microenvironment and is a wellknown premise of spermatogenesis [91-93]. Yi et al. have proved that the accumulation of autophagosome affects the integrity of BTB, which finally contributes to spermatogenesis disturbance, accumulation of damaged mitochondria, and infertility [26]. Therefore, reducing autophagy induced by ROS may become an effective method for preventing male infertility in diabetic patients.

\section{Autophagy Aggravating the Oxidative Damage in Testes}

The autophagy-related protein p62, as a scaffold protein, binds ubiquitinated substrates and aids their aggregation and degradation by macroautophagy [94]. And as a target of autophagy, p62 is constantly controlled by constitutive autophagy [95, 96] (Figure 1). Komatsu and colleagues have also shown that $\mathrm{p} 62$, as an endogenous protein, activates nuclear factor erythroid 2-related factor 2 (Nrf2) by competitive combination of Kelch-like ECH-associated protein 1 (Keap1), a redox-sensitive E3 ubiquitin ligase substrate adaptor, which strictly regulates the intracellular Nrf2 abundance $[97,98]$. Nrf2, a key factor in the cellular antioxidant system, can respond to oxidative stress [99-101]. Under homeostatic conditions, low levels of Nrf2 are primarily maintained by Keap1-mediated proteasomal degradation [102]. Under oxidative stress conditions, Keap1 is oxidized at reactive cysteine residues, resulting in inactivation of Keap1 and stabilization of Nrf2, which then translocates into the nucleus and subsequently binds to antioxidant response elements to promote the expression of downstream cytoprotective proteins that act as scavengers for diabetesinduced free radicals [103-105]. Jain and colleagues also demonstrated that p62 creates a positive feedback loop in the Keap1-Nrf2 pathway and the loop will be broken by the autophagic degradation of p62 [106] (Figure 1). In previous studies, we have found that p62 expression was significantly decreased, Keap1 was significantly increased, and the ratio of nuclear Nrf2 to cytosolic Nrf2 was decreased in the T1D group [107]. We speculate that in the T1D group, when the autophagy is induced by ROS, p62 is degraded and the feedforward loop linking Nrf2 and p62 is broken, which directly results in a decrease in antioxidant capacity and an increase in ROS (Figure 1).

Increased Nrf2 expression can increase the antioxidant capacity of sperm in diabetic patients, which has been confirmed in previous studies. Jiang et al. have shown that sulforaphane may prevent testicular oxidative damage and apoptosis by increasing testicular Nrf2 expression under diabetic condition [108]. Pan et al. have proved that the Nrf2 knockout mice exhibited more significant diabetes-induced loss in testicular weight and sperm count, as well as increased testicular apoptotic cell death compared to wild-type mice, and have demonstrated that Nrf2 plays a critical role in ameliorating diabetic testicular damage [109]. We have also 
shown that resveratrol can attenuate testicular apoptosis in T1D mice via activating Nrf2 through the PI3K/Akt pathway and p62-dependent Keap1 degradation in our previous study [107] (Figure 1). Therefore, increasing the expression of Nrf2 may become an effective method for preventing male infertility.

\section{Conclusion}

As mentioned above, infertility is a common complication of diabetic men, and there is already some evidence to support the role of ROS and autophagy in the pathophysiology of male infertility caused by diabetes. In this review, we elucidate the interaction between ROS and autophagy in diabetic testes via the PI3K/Akt/mTOR signaling pathway and highlight that autophagy induced by ROS aggravates oxidative damage via breaking the feedforward loop linking Nrf2 and p62. Moreover, we have suggested that reducing the production of ROS via decreasing the serum glucose concentration may be effective to treat and prevent male infertility in diabetic patients. And we have also showed that upregulating the Nrf2-Keap1 pathway can increase the ratio of nuclear Nrf2 to cytosolic Nrf2 and enhance the transcription of antioxidant enzymes, such as superoxide dismutase, glutathione peroxidase, and catalase. Additionally, supplementation with nonenzymatic antioxidants such as resveratrol, glutathione, carnitine, pyruvate, vitamin $\mathrm{C}$ (ascorbic acid), and vitamin $\mathrm{E}$ ( $\alpha$-tocopherol) may be effective to augment the scavenging capacity of testes.

Although it has been demonstrated that ROS could promote the formation of autophagy, in turn, autophagy may contribute to aggravate oxidative damage by degrading p62; the internal molecular regulatory mechanisms between ROS and autophagy are complicated in diabetic testicular cells and still need further research.

\section{Abbreviations}

ROS: Reactive oxygen species

SCs: $\quad$ Sertoli cells

BTB: Blood-testis barrier

PI3K: Phosphatidylinositol 3-kinase

Akt: $\quad$ Protein kinase B

mTOR: Mammalian target of rapamycin

DM: Diabetes mellitus

AGEs: Advanced glycation end products

RAGE: Receptors of advanced glycation end products

PUFA: Polyunsaturated fatty acids

$\mathrm{H}_{2} \mathrm{O}_{2}$ : Hydrogen peroxide

LC3B: Light chain 3B

Nrf2: Nuclear factor erythroid 2-related factor 2

Keap1: Kelch-like ECH-associated protein 1.

\section{Conflicts of Interest}

The authors declare no conflicts of interest regarding the publication of this paper.

\section{Acknowledgments}

This study was supported by grants from the National Science Foundation of China (81974227 and 81670221 to YGZ).

\section{References}

[1] Y. Zheng, S. H. Ley, and F. B. Hu, "Global aetiology and epidemiology of type 2 diabetes mellitus and its complications," Nature Reviews Endocrinology, vol. 14, no. 2, pp. 88-98, 2018.

[2] International Diabetes Federation, IDF Diabetes Atlas, DiabetesAtlas, 7th edition, 2019, http://www.diabetesatlas.org/.

[3] J. Ramalho-Santos, S. Amaral, and P. J. Oliveira, "Diabetes and the impairment of reproductive function: possible role of mitochondria and reactive oxygen species," Current Diabetes Reviews, vol. 4, no. 1, pp. 46-54, 2008.

[4] J. Bellver and J. Donnez, "Introduction: Infertility etiology and offspring health," Fertility and Sterility, vol. 111, no. 6, pp. 1033-1035, 2019.

[5] J. Gandhi, G. Dagur, K. Warren et al., "The role of diabetes mellitus in sexual and reproductive health: an overview of pathogenesis, evaluation, and management," Current Diabetes Reviews, vol. 13, no. 6, pp. 573-581, 2017.

[6] I. M. Agbaje, D. A. Rogers, C. M. McVicar et al., "Insulin dependant diabetes mellitus: implications for male reproductive function," Human Reproduction, vol. 22, no. 7, pp. 18711877, 2007.

[7] J. Mulholland, C. Mallidis, I. Agbaje, and N. McClure, "Male diabetes mellitus and assisted reproduction treatment outcome," Reproductive Biomedicine Online, vol. 22, no. 2, pp. 215-219, 2011.

[8] M. G. Alves, A. D. Martins, J. E. Cavaco, S. Socorro, and P. F. Oliveira, "Diabetes, insulin-mediated glucose metabolism and Sertoli/blood-testis barrier function," Tissue Barriers, vol. 1, no. 2, article e23992, 2014.

[9] C. C. Maresch, D. C. Stute, M. G. Alves, P. F. Oliveira, D. M. de Kretser, and T. Linn, "Diabetes-induced hyperglycemia impairs male reproductive function: a systematic review," Human Reproduction Update, vol. 24, no. 1, pp. 86-105, 2018.

[10] S. Soudamani, T. Malini, and K. Balasubramanian, "Effects of streptozotocin-diabetes and insulin replacement on the epididymis of prepubertal rats: histological and histomorphometric studies," Endocrine Research, vol. 31, no. 2, pp. 8198, 2012.

[11] L. Seethalakshmi, M. Menon, and D. Diamond, "The effect of streptozotocin-induced diabetes on the neuroendocrine-male reproductive tract axis of the adult rat," The Journal of Urology, vol. 138, no. 1, pp. 190-194, 1987.

[12] W. R. Scarano, A. G. Messias, S. U. Oliva, G. R. Klinefelter, and W. G. Kempinas, "Sexual behaviour, sperm quantity and quality after short-term streptozotocin-induced hyperglycaemia in rats," International Journal of Andrology, vol. 29, no. 4, pp. 482-488, 2006.

[13] J. Ballester, M. C. Munoz, J. Dominguez, T. Rigau, J. J. Guinovart, and J. E. Rodriguez-Gil, "Insulin-dependent diabetes affects testicular function by FSH- and LH-linked mechanisms," Journal of Andrology, vol. 25, no. 5, pp. 706-719, 2004.

[14] V. Bartak, M. Josifko, and M. Horackova, "Juvenile diabetes and human sperm quality," International Journal of Fertility, vol. 20, no. 1, pp. 30-32, 1975. 
[15] R. S. Padron, A. Dambay, R. Suarez, and J. Mas, "Semen analyses in adolescent diabetic patients," Acta Diabetologica Latina, vol. 21, no. 2, pp. 115-121, 1984.

[16] P. Ranganathan, A. M. Mahran, J. Hallak, and A. Agarwal, "Sperm cryopreservation for men with nonmalignant, systemic diseases: a descriptive study," Journal of Andrology, vol. 23, no. 1, pp. 71-75, 2002.

[17] T. Khamis, A. F. Abdelalim, S. H. Abdallah, A. A. Saeed, N. M. Edress, and A. H. Arisha, "Early intervention with breast milk mesenchymal stem cells attenuates the development of diabetic-induced testicular dysfunction via hypothalamic Kisspeptin/Kiss1r-GnRH/GnIH system in male rats," Biochimica et Biophysica Acta (BBA) - Molecular Basis of Disease, vol. 1866, no. 1, article 165577, 2020.

[18] E. Sajadi, S. Dadras, M. Bayat et al., "Impaired spermatogenesis associated with changes in spatial arrangement of Sertoli and spermatogonial cells following induced diabetes," Journal of Cellular Biochemistry, vol. 120, no. 10, pp. 1731217325, 2019.

[19] Y. P. Jiang, R. J. Ye, J. M. Yang et al., "Protective effects of salidroside on spermatogenesis in streptozotocin induced type-1 diabetic male mice by inhibiting oxidative stress mediated blood-testis barrier damage," Chemico-Biological Interactions, vol. 315, article 108869, 2020.

[20] S. Amaral, A. J. Moreno, M. S. Santos, R. Seica, and J. Ramalho-Santos, "Effects of hyperglycemia on sperm and testicular cells of Goto-Kakizaki and streptozotocin-treated rat models for diabetes," Theriogenology, vol. 66, no. 9, pp. 2056-2067, 2006.

[21] L. Li, J. Tan, Y. Miao, P. Lei, and Q. Zhang, "ROS and autophagy: interactions and molecular regulatory mechanisms," Cellular and Molecular Neurobiology, vol. 35, no. 5, pp. 615-621, 2015.

[22] H. Zhang, X. Kong, J. Kang et al., "Oxidative stress induces parallel autophagy and mitochondria dysfunction in human glioma U251 cells," Toxicological Sciences, vol. 110, no. 2, pp. 376-388, 2009.

[23] R. Scherz-Shouval, E. Shvets, E. Fass, H. Shorer, L. Gil, and Z. Elazar, "Reactive oxygen species are essential for autophagy and specifically regulate the activity of Atg4," EMBO Journal, vol. 26, no. 7, pp. 1749-1760, 2007.

[24] R. Scherz-Shouval and Z. Elazar, "Regulation of autophagy by ROS: physiology and pathology," Trends in Biochemical Sciences, vol. 36, no. 1, pp. 30-38, 2011.

[25] W. Huang, Z. Cao, J. Zhang, Q. Ji, and Y. Li, "Aflatoxin B1 promotes autophagy associated with oxidative stressrelated PI3K/AKT/mTOR signaling pathway in mice testis," Environmental Pollution, vol. 255, article 113317, 2019.

[26] W. E. I. Yi, T. Xiang-Liang, Z. Yu et al., "DEHP exposure destroys blood-testis barrier (BTB) integrity of immature testes through excessive ROS-mediated autophagy," Genes \& Diseases, vol. 5, no. 3, pp. 263-274, 2018.

[27] Y. Wei, X. N. Cao, X. L. Tang et al., "Urban fine particulate matter (PM2.5) exposure destroys blood-testis barrier (BTB) integrity through excessive ROS-mediated autophagy," Toxicology Mechanisms and Methods, vol. 28, no. 4, pp. 302-319, 2017.

[28] Y. Shang, H. Wang, P. Jia et al., "Autophagy regulates spermatid differentiation via degradation of PDLIM1," Autophagy, vol. 12, no. 9, pp. 1575-1592, 2016.
[29] H. Wang, H. Wan, X. Li et al., "Atg7 is required for acrosome biogenesis during spermatogenesis in mice," Cell Research, vol. 24, no. 7, pp. 852-869, 2014.

[30] X. Zhao, W. Xu, J. Wu et al., "Nicotine induced autophagy of Leydig cells rather than apoptosis is the major reason of the decrease of serum testosterone," International Journal of Biochemistry \& Cell Biology, vol. 100, pp. 30-41, 2018.

[31] P. Duan, C. Hu, C. Quan et al., "4-Nonylphenol induces apoptosis, autophagy and necrosis in Sertoli cells: involvement of ROS-mediated AMPK/AKT-mTOR and JNK pathways," Toxicology, vol. 341-343, pp. 28-40, 2016.

[32] Y. J. Lee, J. E. Oh, and S. H. Lee, “Arctigenin shows preferential cytotoxicity to acidity-tolerant prostate carcinoma PC-3 cells through ROS-mediated mitochondrial damage and the inhibition of PI3K/Akt/mTOR pathway," Biochemical and Biophysical Research Communications, vol. 505, no. 4, pp. 1244-1250, 2018.

[33] L. X. Feng, N. Ravindranath, and M. Dym, "Stem cell factor/c-kit up-regulates cyclin D3 and promotes cell cycle progression via the phosphoinositide 3-kinase/p70 S6 kinase pathway in spermatogonia," Journal of Biological Chemistry, vol. 275, no. 33, pp. 25572-25576, 2000.

[34] D. Heras-Sandoval, J. M. Perez-Rojas, J. Hernandez-Damian, and J. Pedraza-Chaverri, "The role of PI3K/AKT/mTOR pathway in the modulation of autophagy and the clearance of protein aggregates in neurodegeneration," Cellular Signalling, vol. 26, no. 12, pp. 2694-2701, 2014.

[35] S. Saiki, Y. Sasazawa, Y. Imamichi et al., "Caffeine induces apoptosis by enhancement of autophagy via PI3K/Akt/mTOR/p70S6K inhibition," Autophagy, vol. 7, no. 2, pp. 176187, 2014.

[36] A. Sapra, S. Vaqar, and P. Bhandari, "Diabetes Mellitus," in StatPearls, StatPearls Publishing StatPearls Publishing LLC, Treasure Island, FL, USA, 2019.

[37] J. Tuomilehto, "The emerging global epidemic of type 1 diabetes," Current Diabetes Reports, vol. 13, no. 6, pp. 795-804, 2013.

[38] J. S. Johansen, A. K. Harris, D. J. Rychly, and A. Ergul, "Oxidative stress and the use of antioxidants in diabetes: linking basic science to clinical practice," Cardiovascular Diabetology, vol. 4, no. 1, p. 5, 2005.

[39] S. Kawahito, H. Kitahata, and S. Oshita, "Problems associated with glucose toxicity: role of hyperglycemia-induced oxidative stress," World Journal of Gastroenterology, vol. 15, no. 33, pp. 4137-4142, 2009.

[40] H. Kaneto, J. Fujii, T. Myint et al., "Reducing sugars trigger oxidative modification and apoptosis in pancreatic betacells by provoking oxidative stress through the glycation reaction," Biochemical Journal, vol. 320, no. 3, pp. 855-863, 1996.

[41] C. Mallidis, I. M. Agbaje, D. A. Rogers et al., "Advanced glycation end products accumulate in the reproductive tract of men with diabetes," International Journal of Andrology, vol. 32, no. 4, pp. 295-305, 2009.

[42] J. L. Wautier and A. M. Schmidt, "Protein glycation - a firm link to endothelial cell dysfunction," Circulation Research, vol. 95, no. 3, pp. 233-238, 2004.

[43] C. Mallidis, I. Agbaje, D. Rogers et al., "Distribution of the receptor for advanced glycation end products in the human male reproductive tract: prevalence in men with diabetes mellitus," Human Reproduction, vol. 22, no. 8, pp. 21692177, 2007. 
[44] W. C. L. Ford, "Regulation of sperm function by reactive oxygen species," Human Reproduction Update, vol. 10, no. 5, pp. 387-399, 2004.

[45] R. J. Aitken, J. S. Clarkson, and S. Fishel, "Generation of reactive oxygen species, lipid peroxidation, and human sperm function," Biology of Reproduction, vol. 41, no. 1, pp. 183197, 1989.

[46] E. de Lamirande and C. Gagnon, "Human sperm hyperactivation and capacitation as parts of an oxidative process," Free Radical Biology \& Medicine, vol. 14, no. 2, pp. 157-166, 1993.

[47] R. J. Aitken, M. Paterson, H. Fisher, D. W. Buckingham, and M. van Duin, "Redox regulation of tyrosine phosphorylation in human spermatozoa and its role in the control of human sperm function," Journal of Cell Science, vol. 108, pp. 20172025, 1995.

[48] S. E. M. Lewis, E. T. Donnelly, E. S. L. Sterling, M. S. Kennedy, W. Thompson, and U. Chakravarthy, "Regulators of sperm function," Molecular Human Reproduction, vol. 2, no. 11, pp. 873-878, 1996.

[49] E. de Lamirande, G. Lamothe, and M. Villemure, "Control of superoxide and nitric oxide formation during human sperm capacitation," Free Radical Biology \& Medicine, vol. 46, no. 10, pp. 1420-1427, 2009.

[50] J. G. Alvarez, J. C. Touchstone, L. Blasco, and B. T. Storey, "Spontaneous lipid peroxidation and production of hydrogen peroxide and superoxide in human spermatozoa superoxide dismutase as major enzyme protectant against oxygen toxicity," Journal of Andrology, vol. 8, no. 5, pp. 338-348, 1987.

[51] R. J. Aitken and J. S. Clarkson, "Cellular basis of defective sperm function and its association with the genesis of reactive oxygen species by human spermatozoa," Journal of Reproduction and Fertility, vol. 81, no. 2, pp. 459-469, 1987.

[52] A. Iwasaki and C. Gagnon, "Formation of reactive oxygen species in spermatozoa of infertile patients," Fertility and Sterility, vol. 57, no. 2, pp. 409-416, 1992.

[53] G. Ricci, A. Catizone, R. Esposito, F. A. Pisanti, M. T. Vietri, and M. Galdieri, "Diabetic rat testes: morphological and functional alterations," Andrologia, vol. 41, no. 6, pp. 361368, 2009.

[54] R. J. Aitken and B. J. Curry, "Redox regulation of human sperm function: from the physiological control of sperm capacitation to the etiology of infertility and DNA damage in the germ line," Antioxidants \& Redox Signaling, vol. 14, no. 3, pp. 367-381, 2011.

[55] A. J. Koppers, G. N. De Iuliis, J. M. Finnie, E. A. McLaughlin, and R. J. Aitken, "Significance of mitochondrial reactive oxygen species in the generation of oxidative stress in spermatozoa," Journal of Clinical Endocrinology \& Metabolism, vol. 93, no. 8, pp. 3199-3207, 2008.

[56] E. Ghanbari, V. Nejati, and M. Khazaei, "Antioxidant and protective effects of royal jelly on histopathological changes in testis of diabetic rats," International Journal of Reproductive Biomedicine, vol. 14, no. 8, pp. 519-526, 2016.

[57] S. La Vignera, R. A. Condorelli, M. Di Mauro et al., "Reproductive function in male patients with type 1 diabetes mellitus," Andrology, vol. 3, no. 6, pp. 1082-1087, 2015.

[58] G. C. Jain and R. N. Jangir, "Modulation of diabetes-mellitusinduced male reproductive dysfunctions in experimental animal models with medicinal plants," Pharmacognosy Reviews, vol. 8, no. 16, pp. 113-121, 2014.
[59] M. Kanter, C. Aktas, and M. Erboga, "Protective effects of quercetin against apoptosis and oxidative stress in streptozotocin-induced diabetic rat testis," Food and Chemical Toxicology, vol. 50, no. 3-4, pp. 719-725, 2012.

[60] Y. G. Wang, Z. G. Zhang, W. Y. Guo et al., "Sulforaphane reduction of testicular apoptotic cell death in diabetic mice is associated with the upregulation of Nrf2 expression and function," American Journal of Physiology-Endocrinology and Metabolism, vol. 307, no. 1, pp. E14-E23, 2014.

[61] B. Shrilatha and Muralidhara, "Early oxidative stress in testis and epididymal sperm in streptozotocin- induced diabetic mice: Its progression and genotoxic consequences," Reproductive Toxicology, vol. 23, no. 4, pp. 578-587, 2007.

[62] A. Agarwal, K. Makker, and R. Sharma, "Clinical relevance of oxidative stress in male factor infertility: an update," American Journal of Reproductive Immunology, vol. 59, no. 1, pp. 2-11, 2008.

[63] X. Wang, R. K. Sharma, S. C. Sikka, A. J. Thomas Jr., T. Falcone, and A. Agarwal, "Oxidative stress is associated with increased apoptosis leading to spermatozoa DNA damage in patients with male factor infertility," Fertility and Sterility, vol. 80, no. 3, pp. 531-535, 2003.

[64] K. Makker, A. Agarwal, and R. Sharma, "Oxidative stress \&amp; male infertility," The Indian Journal of Medical Research, vol. 129, no. 4, pp. 357-367, 2009.

[65] R. J. Aitken, "A free radical theory of male infertility," Reproduction, Fertility and Development, vol. 6, no. 1, pp. 19-23, 1994.

[66] R. J. Aitken, "The Amoroso lecture. The human spermatozoon - a cell in crisis?," Journal of Reproduction and Fertility, vol. 115, no. 1, pp. 1-7, 1999.

[67] A. Agarwal, R. A. Saleh, and M. A. Bedaiwy, "Role of reactive oxygen species in the pathophysiology of human reproduction," Fertility and Sterility, vol. 79, no. 4, pp. 829-843, 2003.

[68] A. Zini, M. S. Gabriel, and A. Baazeem, "Antioxidants and sperm DNA damage: a clinical perspective," Journal of Assisted Reproduction and Genetics, vol. 26, no. 8, pp. 427432, 2009.

[69] P. Blume-Jensen, G. Jiang, R. Hyman, K.-F. Lee, S. O'Gorman, and T. Hunter, "Kit/stem cell factor receptor-induced activation of phosphatidylinositol 3 '-kinase is essential for male fertility," Nature Genetics, vol. 24, no. 2, pp. 157-162, 2000.

[70] R. A. Saxton and D. M. Sabatini, "mTOR Signaling in Growth, Metabolism, and Disease," Cell, vol. 168, no. 6, pp. 960-976, 2017.

[71] L. Bererhi, M. Flamant, F. Martinez, A. Karras, E. Thervet, and C. Legendre, "Rapamycin-induced oligospermia," Transplantation, vol. 76, no. 5, pp. 885-886, 2003.

[72] M. A. Deutsch, I. Kaczmarek, S. Huber et al., "Sirolimus-associated infertility: case report and literature review of possible mechanisms," American Journal of Transplantation, vol. 7, no. 10, pp. 2414-2421, 2007.

[73] D. A. Guertin and D. M. Sabatini, "Defining the role of mTOR in cancer," Cancer Cell, vol. 12, no. 1, pp. 9-22, 2007.

[74] J. T. Busada, B. A. Niedenberger, E. K. Velte, B. D. Keiper, and C. B. Geyer, "Mammalian target of rapamycin complex 1 (mTORC1) is required for mouse spermatogonial differentiation in vivo," Developmental Biology, vol. 407, no. 1, pp. 90-102, 2015.

[75] K. W. Mok, D. D. Mruk, W. M. Lee, and C. Y. Cheng, "Rictor/mTORC2 regulates blood-testis barrier dynamics via its 
effects on gap junction communications and actin filament network," The FASEB Journal, vol. 27, no. 3, pp. 1137-1152, 2012.

[76] C. Schell, O. Kretz, W. Liang et al., "The rapamycin-sensitive complex of mammalian target of rapamycin is essential to maintain male fertility," American Journal of Pathology, vol. 186, no. 2, pp. 324-336, 2016.

[77] H. Xu, L. Shen, X. Chen et al., "mTOR/P70S6K promotes spermatogonia proliferation and spermatogenesis in Sprague Dawley rats," Reproductive Biomedicine Online, vol. 32, no. 2, pp. 207-217, 2016.

[78] T. T. Jesus, P. F. Oliveira, J. Silva et al., "Mammalian target of rapamycin controls glucose consumption and redox balance in human Sertoli cells," Fertility and Sterility, vol. 105, no. 3, pp. 825-833.e3, 2016.

[79] S. Y. T. Li, M. Yan, H. Chen et al., "mTORC1/rpS6 regulates blood-testis barrier dynamics and spermatogenetic function in the testis in vivo," American Journal of PhysiologyEndocrinology and Metabolism, vol. 314, no. 2, pp. E174E190, 2018.

[80] Y. Gao, H. Chen, W. Y. Lui, W. M. Lee, and C. Y. Cheng, "Basement membrane laminin $\alpha 2$ regulation of BTB dynamics via its effects on F-actin and microtubule cytoskeletons is mediated through mTORC1 signaling," Endocrinology, vol. 158, no. 4, pp. 963-978, 2017.

[81] C. J. Lin, T. L. Chen, Y. Y. Tseng et al., "Honokiol induces autophagic cell death in malignant glioma through reactive oxygen species-mediated regulation of the p53/PI3K/Akt $/ \mathrm{m}$ TOR signaling pathway," Toxicology and Applied Pharmacology, vol. 304, pp. 59-69, 2016.

[82] G. J. Shi, J. Zheng, X. X. Han et al., "Lycium barbarum polysaccharide attenuates diabetic testicular dysfunction via inhibition of the PI3K/Akt pathway-mediated abnormal autophagy in male mice," Cell and Tissue Research, vol. 374, no. 3, pp. 653-666, 2018

[83] W. Huang, C. Quan, P. Duan, S. Tang, W. Chen, and K. Yang, "Nonylphenol induced apoptosis and autophagy involving the Akt/mTOR pathway in prepubertal Sprague-Dawley male rats in vivo and in vitro," Toxicology, vol. 373, pp. 4153, 2016.

[84] C. Huang, M. Z. Lin, D. Cheng, F. Braet, C. A. Pollock, and X. M. Chen, "KCa3.1 mediates dysfunction of tubular autophagy in diabetic kidneys via PI3k/Akt/mTOR signaling pathways," Scientific Reports, vol. 6, no. 1, 2016.

[85] S. Sato, S. Kataoka, A. Kimura, and Y. Mukai, "Azuki bean (Vigna angularis) extract reduces oxidative stress and stimulates autophagy in the kidneys of streptozotocin-induced early diabetic rats," Canadian Journal of Physiology and Pharmacology, vol. 94, no. 12, pp. 1298-1303, 2016.

[86] L. Zhang, K. Ding, H. D. Wang, Y. Wu, and J. Xu, "Traumatic brain injury-induced neuronal apoptosis is reduced through modulation of PI3K and autophagy pathways in mouse by FTY720," Cellular and Molecular Neurobiology, vol. 36, no. 1, pp. 131-142, 2016.

[87] Y. Shima, K. Miyabayashi, S. Haraguchi et al., "Contribution of Leydig and Sertoli cells to testosterone production in mouse fetal testes," Molecular Endocrinology, vol. 27, no. 1, pp. 63-73, 2013.

[88] C. J. Murphy and J. H. Richburg, "Implications of Sertoli cell induced germ cell apoptosis to testicular pathology," Spermatogenesis, vol. 4, no. 2, article e979110, 2014.
[89] H. Chen, D. D. Mruk, W. M. Lee, and Y. Cheng, "Regulation of spermatogenesis by a local functional axis in the testis: role of the basement membrane-derived noncollagenous 1 domain peptide," The FASEB Journal, vol. 31, no. 8, pp. 3587-3607, 2017.

[90] D. D. Mruk and C. Y. Cheng, "Sertoli-Sertoli and Sertoligerm cell interactions and their significance in germ cell movement in the seminiferous epithelium during spermatogenesis," Endocrine Reviews, vol. 25, no. 5, pp. 747-806, 2004.

[91] L. Su, Z. Wang, S. Xie et al., "Testin regulates the blood-testis barrier via disturbing occludin/ZO-1 association and actin organization," Journal of Cellular Physiology, vol. 24, 2020.

[92] P. G. Stanton, "Regulation of the blood-testis barrier," Seminars in Cell \& Developmental Biology, vol. 59, pp. 166-173, 2016.

[93] D. D. Mruk and C. Y. Cheng, "The mammalian blood-testis barrier: its biology and regulation," Endocrine Reviews, vol. 36, no. 5, pp. 564-591, 2015.

[94] X. Zhou, W. Hao, H. Shi, Y. Hou, and Q. Xu, "Calcium homeostasis disruption - a bridge connecting cadmiuminduced apoptosis, autophagy and tumorigenesis," Oncology Research and Treatment, vol. 38, no. 6, pp. 311-315, 2015.

[95] M. Komatsu, S. Waguri, M. Koike et al., "Homeostatic levels of p62 control cytoplasmic inclusion body formation in autophagy-deficient mice," Cell, vol. 131, no. 6, pp. 1149$1163,2007$.

[96] G. Bjorkoy, T. Lamark, A. Brech et al., "p62/SQSTM1 forms protein aggregates degraded by autophagy and has a protective effect on huntingtin-induced cell death," Journal of Cell Biology, vol. 171, no. 4, pp. 603-614, 2005.

[97] M. Komatsu, H. Kurokawa, S. Waguri et al., "The selective autophagy substrate p62 activates the stress responsive transcription factor Nrf2 through inactivation of Keap1," Nature Cell Biology, vol. 12, no. 3, pp. 213-223, 2010.

[98] K. Itoh, N. Wakabayashi, Y. Katoh et al., "Keap1 represses nuclear activation of antioxidant responsive elements by Nrf2 through binding to the amino-terminal Neh2 domain," Genes \& Development, vol. 13, no. 1, pp. 76-86, 1999.

[99] J. W. Kaspar, S. K. Niture, and A. K. Jaiswal, "Nrf2:INrf2 (Keap1) signaling in oxidative stress," Free Radical Biology \& Medicine, vol. 47, no. 9, pp. 1304-1309, 2009.

[100] H. Zheng, S. A. Whitman, W. Wu et al., "Therapeutic potential of Nrf2 activators in streptozotocin-induced diabetic nephropathy," Diabetes, vol. 60, no. 11, pp. 3055-3066, 2011.

[101] Q. Ma, "Role of Nrf2 in oxidative stress and toxicity," Annual Review of Pharmacology and Toxicology, vol. 53, no. 1, pp. 401-426, 2013.

[102] D. D. Zhang, "Mechanistic studies of the Nrf2-Keap1 signaling pathway," Drug Metabolism Reviews, vol. 38, no. 4, pp. 769-789, 2008.

[103] S. Ruiz, P. E. Pergola, R. A. Zager, and N. D. Vaziri, “Targeting the transcription factor $\mathrm{Nrf} 2$ to ameliorate oxidative stress and inflammation in chronic kidney disease," Kidney International, vol. 83, no. 6, pp. 1029-1041, 2013.

[104] L. Baird, S. Swift, D. Lleres, and A. T. Dinkova-Kostova, "Monitoring Keap1-Nrf2 interactions in single live cells," Biotechnology Advances, vol. 32, no. 6, pp. 1133-1144, 2014.

[105] A. T. Dinkova-Kostova, W. D. Holtzclaw, R. N. Cole et al., "Direct evidence that sulfhydryl groups of Keap1 are the sensors regulating induction of phase 2 enzymes that protect against carcinogens and oxidants," Proceedings of the 
National Academy of Sciences of the United States of America, vol. 99, no. 18, pp. 11908-11913, 2002.

[106] A. Jain, T. Lamark, E. Sjøttem et al., "p62/SQSTM1Is a target gene for transcription factor NRF2 and creates a positive feedback loop by inducing antioxidant response elementdriven gene transcription," Journal of Biological Chemistry, vol. 285, no. 29, pp. 22576-22591, 2010.

[107] Y. Zhao, W. Song, Z. Wang et al., "Resveratrol attenuates testicular apoptosis in type 1 diabetic mice: role of Akt-mediated Nrf2 activation and p62-dependent Keap1 degradation," Redox Biology, vol. 14, pp. 609-617, 2018.

[108] X. Jiang, Y. Bai, Z. Zhang, Y. Xin, and L. Cai, "Protection by sulforaphane from type 1 diabetes-induced testicular apoptosis is associated with the up-regulation of Nrf2 expression and function," Toxicology and Applied Pharmacology, vol. 279, no. 2, pp. 198-210, 2014.

[109] C. Pan, S. Zhou, J. Wu et al., "NRF2 plays a critical role in both self and EGCG protection against diabetic testicular damage," Oxidative Medicine and Cellular Longevity, vol. 2017, Article ID 3172692, 13 pages, 2017. 